\title{
Constitutively active SARM1 variants that induce neuropathy are enriched in ALS patients
}

A. Joseph Bloom ${ }^{1 *} \mathbb{D}$, Xianrong Mao ${ }^{1}$, Amy Strickland ${ }^{1}$, Yo Sasaki ${ }^{1}$, Jeffrey Milbrandt ${ }^{1 *}$ and Aaron DiAntonio ${ }^{2^{*}}$

\begin{abstract}
Background: In response to injury, neurons activate a program of organized axon self-destruction initiated by the $\mathrm{NAD}^{+}$hydrolase, SARM1. In healthy neurons SARM1 is autoinhibited, but single amino acid changes can abolish autoinhibition leading to constitutively active SARM1 enzymes that promote degeneration when expressed in cultured neurons.
\end{abstract}

Methods: To investigate whether naturally occurring human variants might disrupt SARM1 autoinhibition and potentially contribute to risk for neurodegenerative disease, we assayed the enzymatic activity of all 42 rare SARM1 alleles identified among 8507 amyotrophic lateral sclerosis (ALS) patients and 9671 controls. We then intrathecally injected mice with virus expressing SARM1 constructs to test the capacity of an ALS-associated constitutively active SARM1 variant to promote neurodegeneration in vivo.

Results: Twelve out of 42 SARM1 missense variants or small in-frame deletions assayed exhibit constitutive NADase activity, including more than half of those that are unique to the ALS patients or that occur in multiple patients. There is a > 5-fold enrichment of constitutively active variants among patients compared to controls. Expression of constitutively active ALS-associated SARM1 alleles in cultured dorsal root ganglion (DRG) neurons is prodegenerative and cytotoxic. Intrathecal injection of an AAV expressing the common SARM1 reference allele is innocuous to mice, but a construct harboring $S A R M 1^{V 184 G}$, the constitutively active variant found most frequently among the ALS patients, causes axon loss, motor dysfunction, and sustained neuroinflammation.

Conclusions: These results implicate rare hypermorphic SARM1 alleles as candidate genetic risk factors for ALS and other neurodegenerative conditions.

Keywords: ALS, SARM1, Neurodegeneration, Axon, NAD, Human genetics, Neuropathy

\footnotetext{
* Correspondence: ajbloom@wustl.edu; jmilbrandt@wustl.edu;

diantonio@wustl.edu

${ }^{1}$ Needleman Center for Neurometabolism and Axonal Therapeutics and

Department of Genetics, Washington University School of Medicine in Saint

Louis, St. Louis, MO, USA

${ }^{2}$ Needleman Center for Neurometabolism and Axonal Therapeutics and Department of Developmental Biology, Washington University School of Medicine in Saint Louis, St. Louis, MO, USA
}

(c) The Author(s). 2022 Open Access This article is licensed under a Creative Commons Attribution 4.0 International License, which permits use, sharing, adaptation, distribution and reproduction in any medium or format, as long as you give appropriate credit to the original author(s) and the source, provide a link to the Creative Commons licence, and indicate if changes were made. The images or other third party material in this article are included in the article's Creative Commons licence, unless indicated otherwise in a credit line to the material. If material is not included in the article's Creative Commons licence and your intended use is not permitted by statutory regulation or exceeds the permitted use, you will need to obtain permission directly from the copyright holder. To view a copy of this licence, visit http://creativecommons.org/licenses/by/4.0/. The Creative Commons Public Domain Dedication waiver (http://creativecommons.org/publicdomain/zero/1.0/) applies to the data made available in this article, unless otherwise stated in a credit line to the data. 


\section{Background}

In recent decades, genetic studies have become a principle tool for discovering the molecular pathogenesis underlying neurodegenerative disease. Such investigations have proved especially fruitful for amyotrophic lateral sclerosis (ALS), a rare and devastating motor neuropathy currently linked to over two dozen distinct genetic causes that together explain $\sim 10 \%$ of ALS cases [1]. As with other diseases, discovery of ALS genes was facilitated by technological innovations enabling successively more powerful methods, from family-based linkage studies to genome-wide association studies, and more recently, large exome-sequencing efforts using sophisticated statistical techniques in large case-control groups [2-4]. However, power to detect such associations is naturally attenuated for genes harboring low penetrance variants that contribute to risk without necessarily causing disease, which is particularly relevant given the oligogenic nature of sporadic ALS [5]. This difficulty is compounded when the functional consequences of rare variants cannot be predicted from sequence alone i.e. for genes where protein-truncating alleles are not assumed to be pathogenic [3], and all the more so when variants in the same gene may confer either increased disease risk or protection. In such cases, the power to detect significant associations can only be improved by systematically characterizing all the functional variants in a candidate gene [6]. Here we take such a hypothesis-driven approach to identify potentially pathogenic alleles of SARM1, a key driver of axon degeneration.

Trauma and disease in the nervous system activate an intrinsic axon self-destruction pathway, also known as Wallerian degeneration, which facilitates the orderly clearance of damaged axon segments. This choice between maintaining or actively dismantling axons is primarily determined by the action of SARM1, a TIRcontaining $\mathrm{NAD}^{+}$hydrolase that cleaves $\mathrm{NAD}^{+}$to generate nicotinamide and cyclic ADPR (cADPR), a useful biomarker of SARM1 activity [7]. In healthy neurons, SARM1 is maintained in an autoinhibited state, but injury- or disease-induced depletion of the axon survival factor NMNAT2 activates SARM1 leading to a stereotyped sequence of events beginning with rapid loss of $\mathrm{NAD}^{+}$, followed by loss of ATP, defects in mitochondrial movement and depolarization, influx of calcium, externalization of phosphatidylserine, and ultimately axon fragmentation $[8,9]$. Sarm1 knockout mice are viable and without apparent phenotypes under routine conditions, but are protected against neurodegeneration in models of axotomy, traumatic brain injury, peripheral neuropathy, glaucoma, and retinal degenerative diseases [10-18]. Conversely, mutations that decrease NMNAT2 activity lead to polyneuropathy in both humans and model organisms [19, 20], suggesting that aberrant SARM1 activation has a role in human disease. Furthermore, the recent observation that single point mutations in SARM1 can disrupt enzyme autoinhibition [21-25] led us to speculate that naturally-occurring human variants might similarly dysregulate SARM1 and thereby increase disease risk. ALS was an attractive candidate disease because genetic data from multiple large ALS case-control studies are publicly-available. Notably, polymorphisms in the SARM1 locus have been repeatedly identified by large GWAS of ALS, though the findings were largely discounted due to lack of non-synonymous variants in high linkage disequilibrium and eQTL-effects upon an adjacent gene, POLDIP $2^{26,27}$. ALS also warrants particular attention because peripheral axon degeneration accompanies and may precede motoneuron death during ALS progression [26, 27].

To investigate whether $S A R M 1$ mutations that disrupt the enzyme's autoinhibition are associated with neurogenerative disorders, we sought to identify rare prodegenerative $S A R M 1$ missense variants by biochemically analyzing every rare $S A R M 1$ variant identified in patients and controls from multiple ALS databases. Provocatively, the majority of our strongest candidate variants disrupt SARM1 regulation and confer constitutive activity in vitro. Furthermore, we demonstrate that the enrichment of rare variants found in ALS patients is driven in large part by an enrichment of experimentally-confirmed constitutively active variants. Finally, expression of a constitutively-active SARM1 allele found in three unrelated patients causes an ALS-like phenotype-motor dysfunction, cell death, axon loss and sustained neuroinflammation-when expressed in the mouse spinal cord via intrathecal delivery. We therefore propose that hypermorphic SARM1 mutations are a congenital risk factor for ALS. Because the pathogenic effects of SARM1 activity are not limited to motoneurons, we also hypothesize that rare $S A R M 1$ variation may raise risk for other neurodegenerative conditions such as peripheral neuropathies. While this manuscript was under review for publication, a complementary study using different methods also identified constitutive activity in several of the same ALS-associated rare SARM1 variants reported here [28].

\section{Materials and methods \\ ALS consortia databases}

This study is based on anonymized publicly available human genetic data not associated with demographic or clinical information beyond ALS case status. All assayed $S A R M 1$ polymorphisms were reported in one of three databases, accessed January 2020: Project MinE (http://databrowser.projectmine.com), the University of Massachusetts Medical School Sporadic ALS 
Variant Server (http://als.umassmed.edu/index. php\#SALSbrowser), and the ALS Knowledge Portal (http://alskp.org).

\section{Mice}

Male and female WT and Sarm1 knockout C57BL/6 mice were housed and used under the direction of institutional animal study guidelines at Washington University in St. Louis. The inverted screen test of strength and measurement of grip strength were performed as previously described $[29,30]$. All protocols received institutional IACUC approval.

\section{DRG culture}

Mouse DRG culture was performed as previously described [31]. DRG were dissected from embryonic day 13.5 Sarm1 knockout C57BL/6 or CD1 mouse embryos and cells suspended in growth medium at a concentration of $\sim 7 \times 10^{6}$ cells $/ \mathrm{ml}$ in 96 - well tissue culture plates (Corning) coated with poly-d-Lysine $(0.1 \mathrm{mg} / \mathrm{ml}$; Sigma) and laminin $(3 \mu \mathrm{g} / \mathrm{ml}$; Invitrogen). Lentiviral particles containing SARM1 variants and EGFP were generated as previously described [31]. Lentivirus was added (1-10× $10^{3} \mathrm{pfu}$ ) after $1-2$ days (DIV) and metabolites were extracted or assays were performed at 6-7 DIV. Cell death was quantified by assaying mitochondrial function (MTT assay), as previously described [32].

\section{Automated quantification of Annexin $\mathbf{V}$ staining}

To quantify Annexin V staining, the Alexa Fluor ${ }^{\mathrm{rat}} 568$ conjugate (ThermoFisher) was added to the cultured neurons at a 1:100 dilution four days after viral infection. Bright field and fluorescent images were acquired one hour later using Operetta. Unbiassed image analysis was performed using ImageJ as follows: total axon area was measured from the binary bright field images after subtracting background. For Annexin fluorescent intensity measurement, the fluorescent images were background subtracted and then Annexin positive area was defined using the particle analyzer. Data was reported as the total fluorescent intensity of the Annexin positive area divided by the axon area.

DRG metabolite extraction and metabolite measurement At DIV6, tissue culture plates were placed on ice and culture medium replaced with ice-cold saline $(0.9 \% \mathrm{NaCl}$ in water, $500 \mu \mathrm{l}$ per well). Saline was removed and replaced with $160 \mu \mathrm{l}$ ice cold $50 \% \mathrm{MeOH}$ in water. Solution was transferred to tubes containing $50 \mu \mathrm{l}$ chloroform on ice, shaken vigorously, and centrifuged at $20,000 \mathrm{~g}$ for $15 \mathrm{~min}$ at $4{ }^{\circ} \mathrm{C}$. The clear aqueous phase $(140 \mu \mathrm{l})$ was transferred into microfuge tubes and lyophilized under vacuum. Lyophilized samples were reconstituted with $5 \mathrm{mM}$ ammonium formate $(15 \mu \mathrm{l})$, centrifuged $\left(13,000 \mathrm{~g}, 10 \mathrm{~min}, 4^{\circ} \mathrm{C}\right)$, and $10 \mu \mathrm{l}$ of clear supernatant was analyzed. $\mathrm{NAD}^{+}$and $\mathrm{CADPR}$ were measured using LC-MS/MS as previously described [33].

\section{AAV constructs and virus injections}

AAV particles with a mixture of Php.s and Php.eB capsids [34], containing a human SARM1 gene construct fused to EGFP, under the control of the human synapsin promoter, were produced by the Viral Vector Core of the Hope Center for Neurological Disorders at Washington University in St. Louis. Viral particles were purified by iodixanol gradient ultracentrifugation and virus titers were measured by dot blot. Under light anesthesia with Avertin, $6 \times 10^{11}$ viral genomes were injected intrathecally at L6/S1. Viral expression in mice 12-weeks post injection was confirmed by detecting EGFP expression via immunohistochemical analysis of sectioned DRGs.

\section{Immunohistochemistry, imaging and quantification}

After perfusion with PBS followed by 4\% PFA in PBS, tissues were fixed in 4\% PFA in PBS for $1 \mathrm{~h}$ at room temperature and placed in 30\% sucrose in PBS overnight at $4{ }^{\circ} \mathrm{C}$, then embedded in OCT (Tissue-Tek), frozen on dry ice, and stored at $-80^{\circ} \mathrm{C}$. Longitudinal sections of $6 \mu \mathrm{m}$ or cross-sections of $20 \mu \mathrm{m}$ were obtained using a cryostat and slides were stored at $-20^{\circ} \mathrm{C}$. DRG and nerve slides were post-fixed in cold acetone, then washed with PBS. Spinal cord slides were simply washed three times in PBS. All slides were subsequently blocked with $4 \%$ BSA and 1\% Triton X-100 in PBS and incubated with rat anti-CD68 (1:500; Bio-Rad) and mouse-antiGFP conjugated to Alexa Fluor 488 (1:250; Thermo Fisher Scientific) overnight in the blocking buffer. Slides were then washed, incubated in secondary antibodies (Jackson ImmunoResearch Laboratories), washed, and mounted in Vectashield with DAPI. Slides were imaged using a DMI 4000B confocal microscope (Leica Microsystems) with a $20 \times$ oil objective and DFC 7000-T camera (Leica Microsystems). For quantification, at least four images were measured per animal. CD68-positive cells were counted by a researcher blinded to the images' treatment group. The total CD68-stained area and nerve area in each image was quantified with the particle analyzer in Image J using a uniform threshold.

\section{TUNEL apoptosis detection}

TUNEL was performed as previously described [35]. Slides prepared for immunohistochemistry were thawed then postfixed with $4 \%$ PFA for $10 \mathrm{~min}$ at room temperature, washed thoroughly with $\mathrm{PBS}$, incubated with $10 \mu \mathrm{g} / \mathrm{ml}$ proteinase $\mathrm{K}$ for $15 \mathrm{~min}$ at $37^{\circ} \mathrm{C}$, then washed with PBS. A positive control slide was incubated in DNase $\mathrm{I}(1 \mathrm{U} / \mathrm{ml})$ for $1 \mathrm{~h}$ at $\mathrm{RT}$, then washed with PBS. Slides were then pretreated with TdT buffer (25 
$\mathrm{mm}$ Tris- $\mathrm{HCl}, 200 \mathrm{~mm}$ sodium cacodylate, $0.25 \mathrm{mg} / \mathrm{ml}$ BSA, $1 \mathrm{~mm}$ cobalt chloride, Roche Diagnostics) at RT for $10 \mathrm{~min}$. To perform end-labeling, TdT buffer was combined with terminal deoxynucleotidyl transferase (Roche Diagnostics, 400 U/slide) and Biotin-16-dUTP (Roche Diagnostics, $4 \mu \mathrm{m}$ ) and added to slides for $1 \mathrm{~h}$ at $37^{\circ} \mathrm{C}$. Slides were thoroughly washed with PBS, then blocked for $30 \mathrm{~min}$ with $5 \%$ normal goat serum in PBS with $0.3 \%$ Triton-X, then incubated with Alexa-Fluorconjugated streptavidin (Jackson ImmunoResearch Laboratories) for $30 \mathrm{~min}$ at $37^{\circ} \mathrm{C}$. Slides were washed and mounted in Vectashield with DAPI.

\section{Toluidine blue staining and axon quantification}

Sural, sciatic and tibial nerves were fixed in 3\% glutaraldehyde in 0.1 M PBS, processed and imaged as previously described [13]. Micrographs were stitched using Leica software and axons were counted using ImageJ. To determine axon size distribution and $\mathrm{G}$ ratios of the sciatic nerve, four nonoverlapping areas per cross section were imaged with a $100 \times$ oil objective of a Zeiss Axioskop and photographed with a Hitachi camera. Photographs were analyzed using a previously described binary imaging analysis method [36].

\section{Statistical analysis}

The frequency of constitutively active SARM1 variants in ALS cases vs. controls was compared using Fisher's Exact test. All other comparisons (metabolite measurements, axon density and size, g-ratio, MTT assay, Annexin- $\mathrm{V}$ staining, inverted screen test, grip strength, CD68 staining) were made using two-tailed t-tests. All statistics were calculated using the $\mathrm{R}$ software package. All data is available upon request.

\section{Results}

\section{Enrichment of rare SARM1 variants in ALS patients}

The discovery of single point mutations in SARM1 that confer constitutive NADase activity leading to degeneration of cultured neurons [10, 22, 23, 37] prompted us to postulate that rare human gain-of-function SARM1 variants might increase risk for neurodegenerative disease. ALS was of particular interest because of the large-scale axon loss that occurs over the course of the disease [26, 27]. To explore this hypothesis, we chose to investigate SARM1 variants found in ALS patients, a population where an abundance of whole exome and genome data is available.

We identified a total of 30 rare SARM1 coding variants (missense and small in-frame deletions with allele frequencies $<0.01 \%$ in all gnomAD populations [38]) occurring exclusively in ALS patients, culled from three large publicly-accessible ALS consortia databases that include 8507 cases in total as of January $2020^{41-43}$ (Table 1). All subjects harboring rare SARM1 variants are heterozygotes. Despite being extremely rare, eight of these variants were each found in two or three ALS patients. We further identified twelve rare SARM1 coding variants that occur in control individuals from these databases. Altogether, rare variants occur 45 times in 8507 ALS cases and 15 times in 9671 controls (Table 1), representing a 3.4-fold enrichment of rare SARM1 variants in ALS patients.

\section{SARM1 variants found in ALS patients exhibit constitutive $\mathrm{NAD}^{+}$hydrolase activity}

To investigate whether the rare SARM1 variants in ALS patients disrupt autoinhibition, we assayed the $\mathrm{NAD}^{+}$ hydrolase activities of the mutant enzymes encoded by these variants. We prioritized the variants and first tested a) those identified in multiple ALS patients but not in healthy controls and b) those unique to ALS patients (i.e. not reported in any prior human study as of January 2020). These 15 SARM1 variants (Fig. 1A, Table 1) account for $51 \%(20 / 39)$ of rare SARM1 variants in ALS patients genotyped in the three large ALS databases we investigated. To examine the properties of these mutants, we tested them in Sarm1 ${ }^{-/-}$mouse dorsal root ganglion DRG neurons. We prepared lentiviruses for the 15 SARM1 mutant constructs, infected $\mathrm{Sarm}^{-/-}$neurons, and assessed their $\mathrm{NAD}^{+}$hydrolase activity. As validated in our prior publication [21], the ratio of the SARM1-specific biomarker cADPR [33] over its parent metabolite $\mathrm{NAD}^{+}$is the best measure of relative SARM1 activity because cADPR production is specific to SARM1, while $\mathrm{NAD}^{+}$concentration is affected by other cellular processes. Moreover, because $\mathrm{NAD}^{+}$is the limiting substrate, the ratio of the two molecules accounts for both SARM1 enzymatic activity and substrate depletion. Measuring cADPR $/ \mathrm{NAD}^{+}$, eight of these variants were determined to be constitutively active, i.e. their baseline levels of $\mathrm{NAD}^{+}$were significantly decreased and levels of cADPR and the ratio of $\mathrm{cADPR} / \mathrm{NAD}^{+}$were significantly increased in neurons expressing these mutant constructs (Fig. 1). Tellingly, all but one of these constitutively active variants reside in the autoinhibitory ARM domain. By contrast, SARM1 $1^{\mathrm{P} 332 \mathrm{Q}}$, the only relatively common variant found in any gnomAD population (1.1\% in Europeans) is not constitutively active (Fig. 1). We confirmed that the measured differences in $\mathrm{NAD}^{+}$ hydrolase activity were not due to differences in construct expression levels using expression of the EGFP also included in the construct (Additional Files 1 and 2). Indeed, the strongest constitutively active mutant constructs were also associated with the weakest EGFP expression (Fig. 1 and Additional Files 1 and 2), a phenomenon we and others observe routinely [28], which we attribute to reduced protein translation due to $\mathrm{NAD}^{+}$depletion in the infected cells. Thus, we are likely 
Table 1 Rare SARM1 missense variants and in-frame deletions found in ALS patients

\begin{tabular}{|c|c|c|c|c|c|c|}
\hline \multirow[t]{2}{*}{ Variant } & \multirow[t]{2}{*}{ rsID } & \multicolumn{3}{|c|}{ Percent Minor Allele Frequency ${ }^{a}$} & \multicolumn{2}{|c|}{ Number of occurrences } \\
\hline & & African & East Asian & European $^{\mathrm{b}}$ & ALS patients & Controls \\
\hline \multicolumn{7}{|c|}{ Constitutively active } \\
\hline$\Delta 226-232$ & rs782325355 & 0 & 0 & 0.01 & $2^{d}$ & 0 \\
\hline$\Delta 249-252$ & & 0 & 0 & 0 & $1^{d}$ & 0 \\
\hline V184G & rs71373646 & 0.007 & 0.006 & 0 & $3^{e}$ & 0 \\
\hline G206S & rs1555585199 & 0 & 0 & 0 & $2^{e}$ & 0 \\
\hline L223P & & 0 & 0 & 0 & $1^{d}$ & 0 \\
\hline R267W & rs1 1652384 & 0 & 0 & 0.001 & $1^{e}$ & 0 \\
\hline V331E & rs1555585331 & 0 & 0 & 0 & $1^{d}$ & 0 \\
\hline E340K & rs781854217 & 0 & 0 & 0.003 & $1^{d}$ & 0 \\
\hline C482Y & & 0 & 0 & 0 & 0 & $1^{\mathrm{d}}$ \\
\hline T385A & & 0 & 0 & 0 & $1^{\mathrm{d}}$ & 0 \\
\hline T502P & rs782421919 & 0 & 0 & 0.006 & $2^{d, f}$ & 0 \\
\hline E693D & rs782331635 & 0 & 0 & 0.005 & 0 & $2^{f}$ \\
\hline \multicolumn{7}{|c|}{ Not constitutively active } \\
\hline V112l & rs1032963037 & 0 & 0 & $0^{c}$ & $1^{\mathrm{d}}$ & 0 \\
\hline A240E & rs1449836804 & 0 & 0 & 0.004 & $1^{d}$ & 0 \\
\hline R244S & & 0 & 0 & 0 & $1^{d}$ & 0 \\
\hline A250T & rs1555585243 & 0 & 0.06 & 0 & $1^{d}$ & 0 \\
\hline A275V & rs376587698 & 0 & 0 & 0.006 & $1^{\mathrm{d}}$ & 0 \\
\hline $\mathrm{R} 310 \mathrm{H}$ & rs369186722 & 0 & 0 & 0.01 & $1^{d}$ & 0 \\
\hline A341V & rs373458416 & 0 & 0 & 0.01 & $2^{d}$ & 0 \\
\hline R403P & rs782706244 & 0 & 0 & 0.0009 & $1^{\mathrm{f}}$ & 0 \\
\hline Y429F & & 0 & 0 & 0 & 0 & $1^{\mathrm{d}}$ \\
\hline E431G & rs1555585662 & 0 & 0 & 0.001 & $1^{d}$ & 0 \\
\hline R465T & & 0 & 0 & 0 & $1^{\mathrm{d}}$ & 0 \\
\hline N478S & rs1555585804 & 0 & 0 & 0.001 & 0 & $1^{f}$ \\
\hline D483E & rs377210302 & 0 & 0 & 0.002 & 0 & $1^{\mathrm{f}}$ \\
\hline R484C & rs1555585809 & 0 & 0 & 0.0009 & $1^{d}$ & 0 \\
\hline A488E & rs782228906 & 0.004 & 0 & 0.02 & $2^{d}$ & 0 \\
\hline V518L & rs782106973 & 0 & 0 & $0^{c}$ & $3^{d}$ & 0 \\
\hline S558N & & 0 & 0 & 0 & 0 & $1^{d}$ \\
\hline R569C & rs571724138 & 0 & 0 & 0.005 & $1^{\mathrm{d}}$ & 0 \\
\hline R570Q & rs539229444 & 0 & 0.008 & 0.005 & $1^{\mathrm{e}}$ & 0 \\
\hline 1593T & rs782196205 & 0 & 0.005 & 0.002 & 0 & $1^{f}$ \\
\hline E604K & rs782398426 & 0.02 & 0.01 & 0.005 & 0 & $1^{f}$ \\
\hline M612V & rs782321764 & 0 & 0 & 0.004 & 0 & $2^{f}$ \\
\hline $\mathrm{R} 615 \mathrm{H}$ & rs782753946 & 0.008 & 0 & 0.004 & $2^{f}$ & $1^{\mathrm{d}}$ \\
\hline D637Y & rs 1451417529 & 0 & 0 & 0 & $1^{\mathrm{d}}$ & 0 \\
\hline A646S & rs782676389 & 0 & 0 & 0.0008 & $1^{d}$ & 0 \\
\hline V654M & rs782225125 & 0 & 0 & 0.002 & 0 & $2^{f}$ \\
\hline M672V & rs782774927 & 0 & 0 & 0.004 & $2^{e, f}$ & 0 \\
\hline S684F & rs782256561 & 0.004 & 0 & 0.004 & $1^{f}$ & 0 \\
\hline R697C & rs372946020 & 0.004 & 0 & 0.003 & $3^{f}$ & $1^{f}$ \\
\hline R702C & rs781850558 & 0 & 0.005 & 0.002 & $1^{f}$ & 0 \\
\hline
\end{tabular}

anomAD v2

non-Finnish

${ }^{\mathrm{V}} 518 \mathrm{~L}=0.009 \%$ and $\mathrm{V} 112 \mathrm{I}=0.001 \%$ in gnomAD v3 non-Finnish Europeans

dMinE [37]

eALS Variant Server [39]

fALS Knowledge Portal [38] 
underestimating the relative activity of constitutively active alleles. We also saw similar activity when mutant SARM1 constructs were expressed in wildtype mouse DRG neurons (i.e. in the presence of endogenous wildtype SARM1) (Additional File 3).

Encouraged by these results, we assayed the activities of an additional 15 rare missense variants. These were considered poorer candidates because each is observed in only a single ALS patient and they are not unique to the patients as they are also found in the gnomAD database. Among these, we identified two additional constitutively active variants (Table 1$)$. In total, $40 \%(4 / 10)$ of the SARM1 variants with constitutive $\mathrm{NAD}^{+}$hydrolase activity occur in multiple ALS patients.

\section{Enrichment of constitutively-active SARM1 variants in ALS patients}

In order to determine whether constitutively active SARM1 variants occur significantly more frequently among ALS patients, we further assayed the activities of all twelve rare SARM1 missense variants reported among 9671 healthy control individuals included in the large publicly-accessible ALS consortia databases (Table 1). Among these, two variants (one of which occurred twice) demonstrated significantly greater enzymatic activity than the reference allele of SARM1, although notably they are the weakest variants that we define as constitutively active (see cADPR/NAD ${ }^{+}$ratio in Fig. 1). In total, 3 of 9671 controls versus 15 of 8507 cases carry a constitutively active $S A R M 1$ variant, representing a significant 5.7-fold enrichment among cases (Fisher's Exact $p=0.0032$ ).

\section{Constitutively active SARM1 variants found in ALS patients promote degeneration of cultured neurons}

The structure of SARM1 in its autoinhibited state, as determined by cryo-EM, indicates the protein exists as a compact octamer with its TIR NADase domains isolated from one another, preventing the TIR oligomerization necessary for enzymatic activation. Mutational analyses identified five distinct and non-redundant interfaces required for autoinhibition that support both intramolecular and intermolecular interactions [24]. Point mutants that disrupt any of these interfaces result in dysregulation of SARM1 activity and this constitutive SARM1 activity promotes the degeneration of cultured neurons $[10,22,23,37]$. As in those prior studies, we also recognized changes in cell morphology consistent with cell death in Sarm1 $1^{-/}$mouse DRG neurons expressing constitutively active variants and chose to quantify cell death and degeneration for two of the variants, SARM1 $1^{V 184 G}$ and SARM1 $1^{\triangle 226-232}$. These two variants were chosen to assay because they occurred in three and two independent ALS patients respectively (Table 1). The mutant enzymes were expressed in Sarm1 ${ }^{-/-}$DRG neurons and degeneration was measured by two methods. Fluorescently-labeled Annexin V, which binds to phosphatidylserine, was used to determine whether the expression of either variant construct significantly compromises axon health. Annexin V binding is a useful proxy for axon health as neurites undergoing Wallerian degeneration expose phosphatidylserine on their extracellular surfaces similarly to apoptotic cells $[9,40]$. Neuronal death was quantified using an oxidoreductase activity assay, a common measure of cell viability. Both assays demonstrated that both ALS-associated SARM1 variants produced a significant degenerative effect relative to the common reference allele (Fig. 2).

While these variants exhibit constitutive $\mathrm{NAD}^{+}$hydrolase activity, it is formally possible that they mediate their pro-degenerative effects via a distinct toxic mechanism. To investigate this alternate hypothesis, we generated constructs containing two mutations, either of the ALS-associated variants, i.e. SARM1 $1^{V 184 G}$ or SARM1 ${ }^{\Delta 226-232}$, together with E642A, a point mutation in the TIR domain that disrupts the catalytic glutamate required for SARM1 $\mathrm{NAD}^{+}$hydrolase activity and axon degeneration [23, 41-44]. In both cases, introducing E642A abolishes enzymatic activity and the detrimental effects of the constructs on cell body and axon health (Fig. 2). Hence, these ALS patient-derived SARM1 variants promote degeneration via loss of autoinhibition and resulting constitutive $\mathrm{NAD}^{+}$hydrolase activity.

\section{The ALS-associated SARM $1^{\text {V184G }}$ variant induces motor impairment, peripheral axon loss and neuroinflammation in mice}

To test whether rare ALS-associated SARM1 variants can promote neurodegeneration in vivo, AAV viral vectors were administered intrathecally to male and female six-week old wild-type mice, expressing either the common human allele of SARM1 (the reference allele) or SARM1 $1^{\text {V184G }}$. V184G was chosen to examine in vivo because it was the rare variant that occurred most frequently among ALS patients, found in three independent subjects. In these constructs, each SARM1 protein was fused to EGFP and expressed under the control of the human synapsin promoter. AAV viruses were produced using a mixture of PHP. S and PHP.eB serotype capsids (both derived from AAV9 [34]) in order to infect neurons in the spinal cord and DRGs.

Animals injected with AAV expressing the common SARM1 allele had no discernible behavioral phenotypes. By contrast, those injected with AAV-SARM1 $1^{V 184 G}$ exhibited motor impairment. Two of the mice rapidly progressed to full limb paralysis 3-4 days after injection. Other animals injected with SARM1 ${ }^{V 184 G}$ (7/9) displayed less dramatic motor deficits characterized by significant 


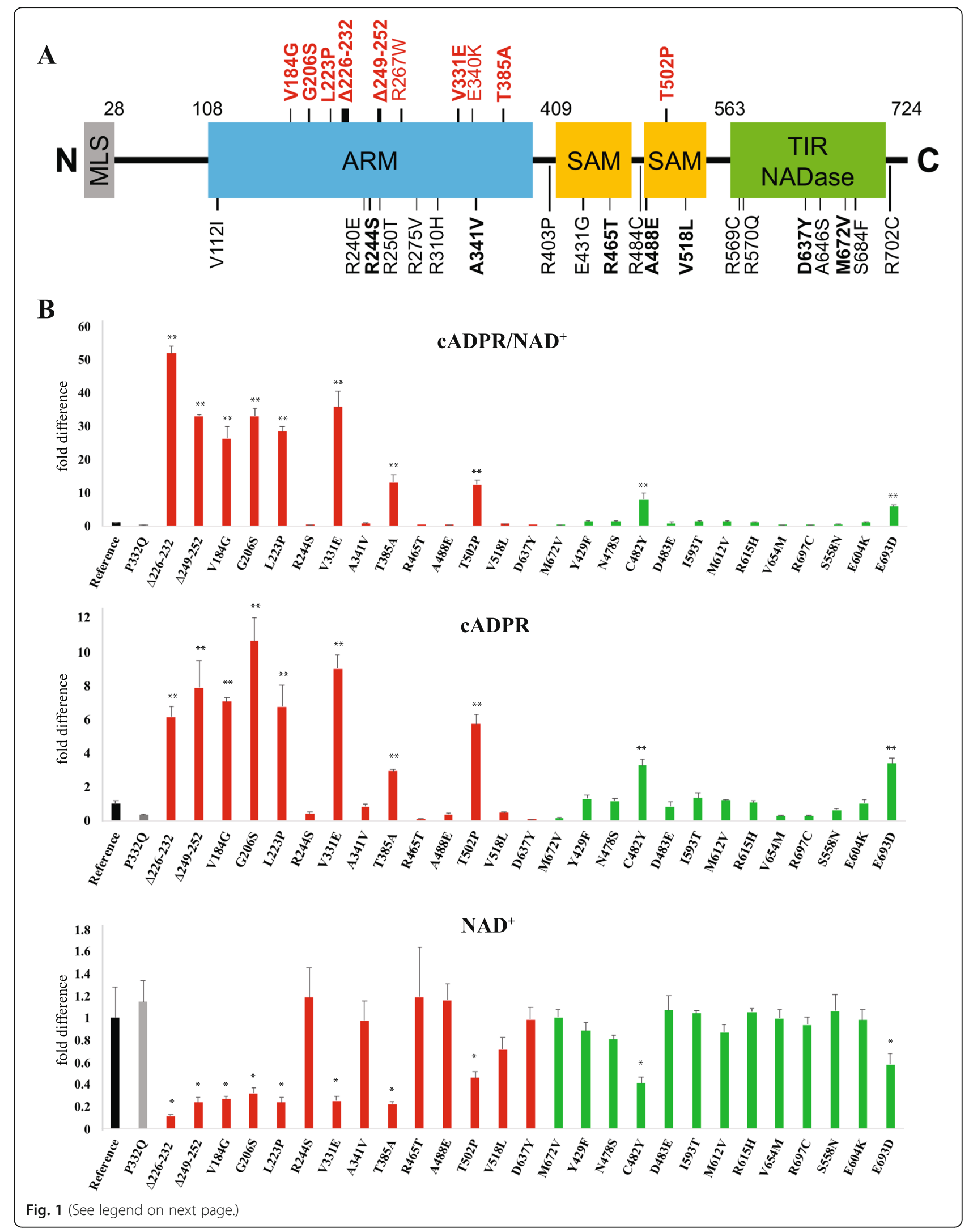


(See figure on previous page.)

Fig. 1 Identification of dysregulated SARM1 variants found in ALS patients. (A) Schematic representation of the domain structure of SARM1 marked with every rare variant found in ALS patients. Constitutively-active variants are indicated above in red. Bold variants were prioritized because they were identified in multiple ALS patients or were unique to ALS patients. $\Delta$ indicates an in-frame deletion. MLS, mitochondrial localization signal; ARM, HEAT/Armadillo motif; SAM, sterile alpha motif; TIR, Toll/interleukin-1 receptor homology domain. (B) The ratio of cADPR/ $\mathrm{NAD}^{+}, \mathrm{CADPR}$, and NAD levels from cultured Sarm 1 ${ }^{-1-}$ DRG neurons infected with human SARM1 constructs carrying every rare variants identified in multiple ALS patients or unique to ALS patients (red), every rare variant found in controls (green), and the most common SARM1 variant, P332Q (gray), relative to the common reference human genome allele of SARM1 (black). ${ }^{*} p<0.05 ;{ }^{* *} p<0.0005$ difference from reference allele. We demonstrate that strongly constitutively active variants predominate among those SARM1 variants identified in multiple ALS patients or unique to ALS patients, while the only two significantly active variants discovered in controls are comparatively weak

muscle weakness as measured by the inverted screen assay (Fig. 3) or hindlimb grip strength (Additional File 4). These deficits were detected within 3 weeks of injection and did not progress significantly during the 12-week observation period.

To characterize the neurodegeneration caused by $S A R M^{V 184 G}$ expression, the intrathecally injected mice were examined for evidence of axon degeneration and neuron loss. We examined the two mice that became rapidly paralyzed and the other mice with less severe disease as separate cohorts because of the difference in phenotype. Unfortunately, because we do not see EGFP expression with any construct until at least a week post- infection, we were unable to use EGFP to confirm expression in mice sacrificed early. In the spinal cords of the paralyzed mice, there was clear evidence of cell death around the ependymal canal as detected by TUNEL staining (Fig. 4, Additional File 5). Neuroinflammation was observed throughout the spinal cord of these mice as evidenced by prevalent staining for CD68, a marker of activated macrophages (Fig. 4). Neither of these phenotypes were observed in animals injected with the common SARM1 allele construct (Fig. 4). In this cohort, levels of CD68 staining in sciatic nerves did not differ between mice injected with $S A R M 1^{V 184 G}$ and the control SARM1 AAV (Additional File 5), nor did any of the mice

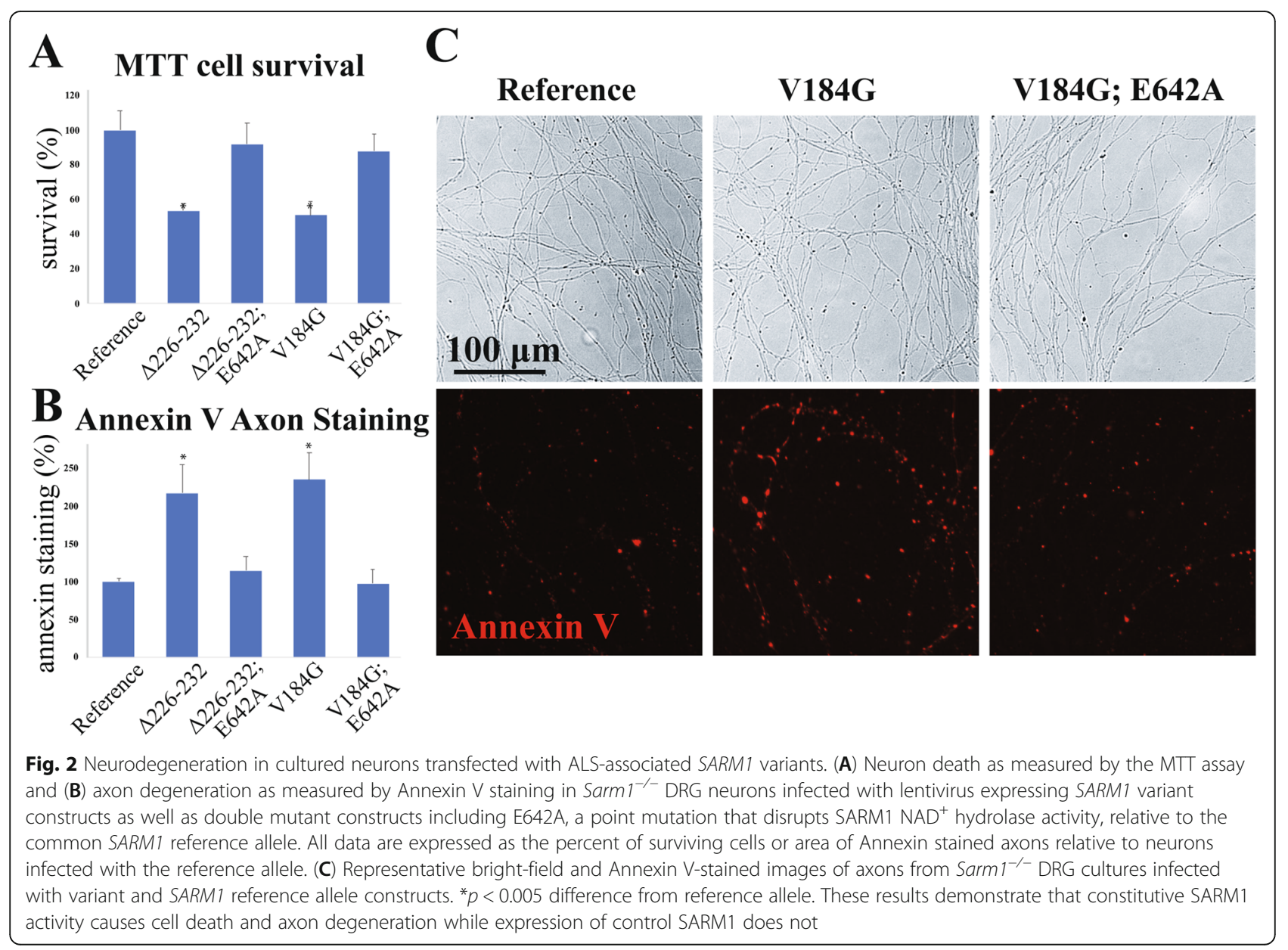




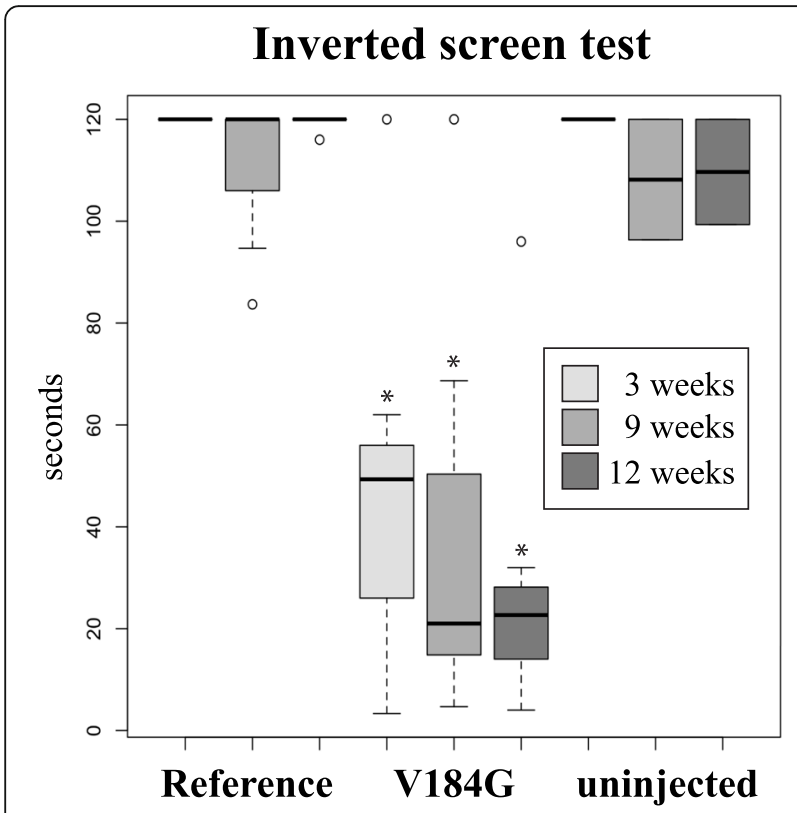

Fig. 3 Motor dysfunction in mice injected intrathecally with a SARM $1^{V 184 G}$ AAV construct. Average time suspended from an inverted screen (maximum 120 s) for C57BL/6 mice injected with a human SARM1 reference allele $(n=8)$ or SARM $1^{V 184 G}(n=7)$ AAV compared to uninjected controls $(n=3) 3,9$ and 12 weeks postinjection. ${ }^{*} \mathrm{p}<0.005$ difference from both the reference allele and uninjected controls. These results demonstrate that infection with SARM ${ }^{V 184 G}$ causes persistent motor dysfunction while the control SARM1 construct does not

display significant myelin defects or vacuolization in the sural, sciatic or tibial nerves 3-4 days post-infection (data not shown).

The mice treated with $S A R M 1^{V 184 G}$ that displayed a less severe behavioral response were sacrificed twelve weeks post-injection. Expression of viral constructs in these mice was confirmed using the presence of EGFP in the DRGs and were similar for each construct (Additional Files 6 and 7). Pathological inspection of their spinal cords revealed no evidence of ongoing apoptosis or elevated CD68 staining (Fig. 5). Their peripheral nerves, however, contain almost 10-fold more CD68positive macrophages than those treated with the control SARM1 allele (Fig. 5). Macrophages increase in size upon activation [45], and the $S A R M 1^{V 184 G}$-infected mice also have a 1.6-fold greater CD68-stained area per cell than do control mice, yielding a 15.2-fold difference in total CD68-stained area. Hence, neuronal expression of SARM $1^{V 184 G}$ triggers an elevated inflammatory response in peripheral nerves that persists for at least twelve weeks after treatment [46].

The average fiber densities in the peripheral nerves of the SARM1 $1^{V 184 G}$-injected mice are also lower than those injected with AAV expressing the common human allele, demonstrating that expression of the constitutively active SARM1 promotes axon loss. The number of axons were counted in the sural, sciatic and tibial nerves. In both the sural and sciatic nerves, the density of axons is significantly lower $(p<0.001)$, a trend that is evident in the tibial nerve but that did not reach statistical significance (Fig. 5). The average axon size and extent of myelination (g-ratio) does not differ significantly between the variant and common SARM1 allele treated animals (Fig. 5, $p>0.1, n=16$ ). Myelin defects and vacuolization are not observed in these nerves, indicating a lack of ongoing axon loss. The lack of axon defects at twelve weeks is consistent with the early, but stable, deficits in motor function observed in mice receiving the SARM1 $1^{V 184 G}$ virus (Fig. 3). We interpret these data as evidence that a subset of neurons-those infected with virus and sufficiently susceptible to SARM1-dependent degeneration-lost their axons before three weeks, while others, including uninfected neurons, remained healthy and functional up to twelve weeks. Inter-animal differences in motor dysfunction severity likely reflect variability in infection efficiency.

\section{Discussion}

SARM1, the chief axon executioner, directs the ordered elimination of damaged axons, a process called Wallerian degeneration. In healthy axons, this potent $\mathrm{NAD}^{+}$ hydrolase is maintained in an autoinhibited state, waiting to be activated by injury or disease. SARM1 activation leads to a rapid loss of $\mathrm{NAD}^{+}$, metabolic catastrophe and subsequent axon fragmentation $[7,9]$. Due to its central role in programmed axon destruction, prior investigations of SARM1 vis-à-vis disease have focused on inhibiting the pathway to block degeneration downstream of specific injury and disease processes [10-16]. This is the first study to address genetically-determined gain-offunction SARM1 activity as a candidate disease mechanism in itself, building upon the discovery of engineered mutations that disrupt SARM1 autoinhibition and promote neurodegeneration in vitro [21-25]. Provocatively, we find that many rare SARM1 variants found in ALS patients also lack normal autoinhibition, and that such an allele induces neurodegeneration and neuroinflammation when expressed in the mouse nervous system.

A number of prior findings are consistent with the hypothesis that SARM1 activity contributes to pathological neurodegeneration, particularly in ALS. First, noncoding variants in the SARM1 locus were repeatedly detected in genome-wide association studies of ALS, though a causal link has been largely discounted [47, 48]. Second, aggregation of TDP-43, a hallmark of most ALS (excluding SOD1-ALS), results in the reduced expression of STMN2, a key axon survival factor that inhibits SARM1-mediated axon destruction [49-52]. Finally, Sarm1 loss suppresses neurodegenerative 

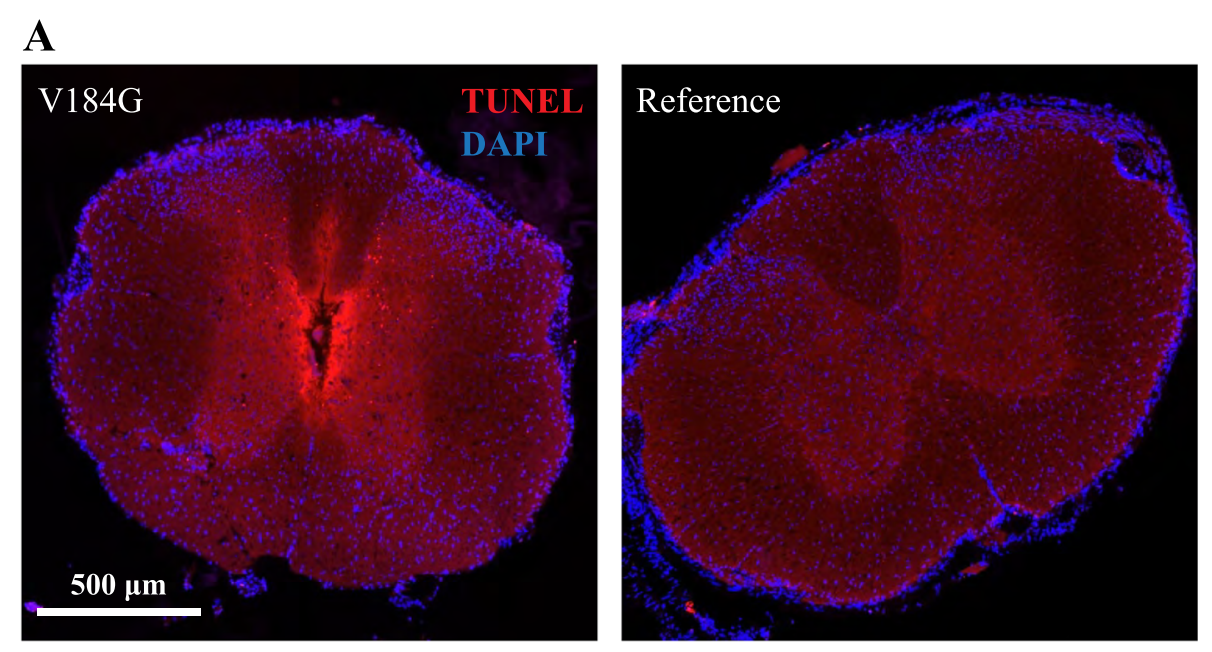

B
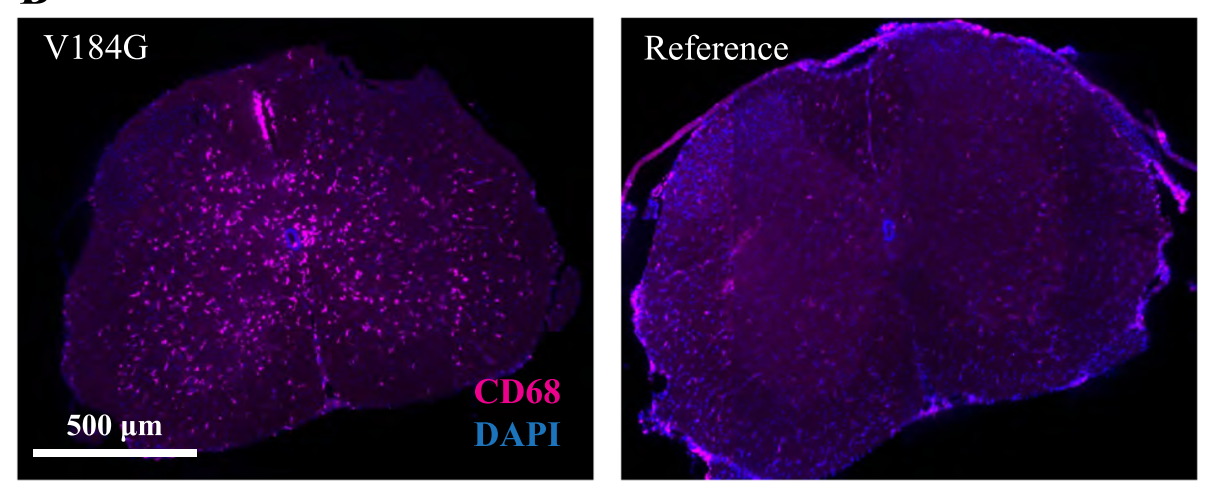

Fig. 4 Rapid cell death and neuroinflammation in mice injected intrathecally with a SARM ${ }^{V 184 G}$ AAV construct. This figure demonstrates anatomical findings from the small subset of mice with rapid onset pathology. (A) Representative images of spinal cord sections stained with DAPI and the apoptosis marker TUNEL from mice 2 days after injection with a SARM1 $1^{V 184 G}$ or SARM1 human reference allele construct. (B) Representative images of spinal cord stained with DAPI and the macrophage marker anti-CD68 from mice 2 days after injection with a $S A R M 1^{V 184 G}$ or reference allele construct. These images demonstrate that mice that become paralyzed shortly after injection with SARM $1^{V 184 G}$ exhibit cell death and activated macrophages in their spinal cords while mice injected with the control SARM1 construct do not

phenotypes in many injury and disease models [10-18], including a mouse that expresses pathogenic human TDP-43 [53]. However, this suppression does have limits, as Sarm1 deletion does not ameliorate ALS symptoms in the $S O D 1^{G 93 A}$ mouse model [30].

The hypothesis that naturally-occurring SARM1 polymorphisms could disinhibit normal enzyme activity and predispose to degenerative disease led us to examine exome sequence data from several well-annotated studies of ALS $[39,54,55]$. We discovered multiple rare SARM1 variants in these patients that result in constitutive activity due to loss of autoinhibition, and consequently promote neuron death and axon degeneration in vitro. Importantly, a single point-mutation that disrupts SARM1 catalytic activity is sufficient to negate the prodegenerative effects of these ALS-associated variants, demonstrating the degenerative phenotype requires SARM1 $\mathrm{NAD}^{+}$hydrolase activity and is not due to a non-specific mechanism such as the toxic aggregation of a misfolded protein. Expression in the mouse CNS of a SARM1 construct harboring one such variant causes motor dysfunction and sustained neuroinflammation, abnormalities not observed in mice treated with the common human reference allele of SARM1. The mechanism by which constitutive activity would predispose to neurodegeneration appears straightforward. Low $\mathrm{NAD}^{+}$ is a death sentence for energy-hungry neurons and is associated with both disease and aging-related functional defects $[56,57]$. Indeed, $\mathrm{NAD}^{+}$augmentation ameliorated ALS symptoms in a small clinical trial [58]. We speculate that the contrast between virus-infected mice that rapidly display severe degenerative phenotypes, and human ALS patients who are typically diagnosed only after several decades of life, likely reflects the difference in SARM1 expression-i.e. viral over-expression precipitates abrupt metabolic catastrophe in this model, 

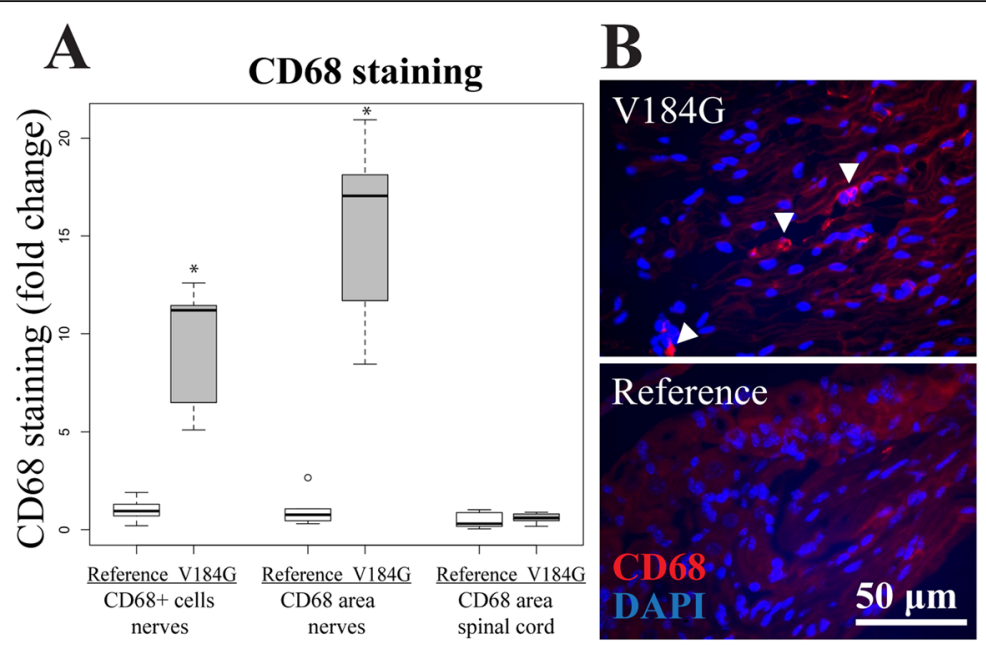

C
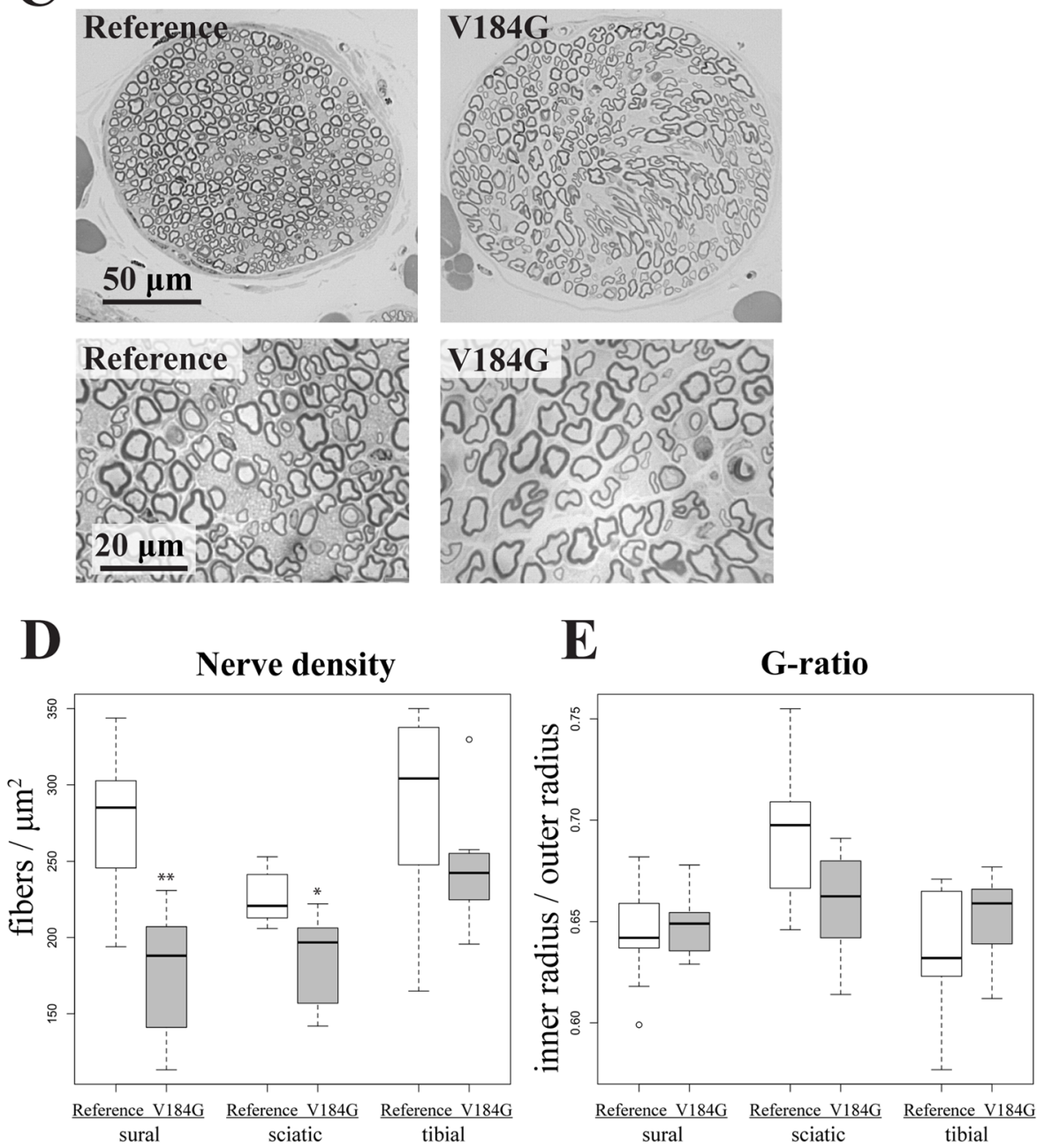

Fig. 5 (See legend on next page.) 
(See figure on previous page.)

Fig. 5 Persistent neuroinflammation and axon loss in mice injected intrathecally with a SARM $7^{V 184 G}$ AAV construct. This figure demonstrates anatomical findings from the larger subset of mice with slower onset pathology. (A) The normalized average number of cells stained by the macrophage marker anti-CD68 in nerve, and the average percent area of total anti-CD68 staining in nerve and in spinal cord sections, from C57BL/6 mice injected with a SARM1 ${ }^{V 184 G}(n=7)$ AAV construct relative to those injected with a human SARM1 reference allele construct $(n=8)$ 12 weeks post-injection; ${ }^{*} p<10^{-4}$ difference from reference allele. (B) Representative images of sural nerve stained with DAPI and anti-CD68 from mice 12 weeks after injection with a SARM1 ${ }^{V 184 G}$ or reference allele construct. White arrows indicate CD68-positive activated macrophages. (C) Representative images of toluidine blue stained sural nerve sections. (D) Average fibers per cross-sectional $\mu m^{2}$ in sural, sciatic and tibial nerves from mice 12 weeks after injection with a SARM $7^{V 184 G}\left(n=7\right.$ mice) or reference allele construct $(n=8) ;{ }^{*} p<0.05$, ${ }^{* *} p<0.001$. (E) Average g-ratio (ratio between the inner and outer myelin sheath) for axons in the sural, sciatic and tibial nerves from mice 12 weeks after injection with a $S A R M 1^{V 184 G}$ or reference allele construct. These analyses demonstrate that mice injected with SARM $7^{V 184 G}$ exhibit axon loss and persistent neuroinflammation in their nerves 12 weeks after infection, compared to mice injected with a control SARM1 construct

whereas chronic suboptimal $\mathrm{NAD}^{+}$levels lead to gradual motoneuron attrition in patients. Hence, our studies demonstrate what activating SARM1 mutations can do in an animal model, but assessing whether such mutations model ALS will require generation of germline mutations in Sarm1 that mimic the human variants.

Sophisticated statistical methods and next-generation sequencing have allowed the contribution of rare variation to disease risk to be addressed using techniques that identify genes containing an excess of presumably deleterious rare variants among cases relative to healthy controls [4]. However, because SARM1 is a switch that may be pushed either 'off' or 'on', the consequence of any genetic perturbation defies simple assumptions. Extensive structure/function analysis has shown that single point mutations in SARM1 can 1) abolish NADase function [41], 2) create a neuroprotective dominant negative $[59], 3)$ alter the enzyme's sensitivity to regulation by NMN and $\mathrm{NAD}^{+}[21,22]$, or 4 ) disrupt its autoinhibitory domain leading to pro-degenerative constitutive activity [24]. Indeed, altering a specific residue by substituting different amino acids at the same position can confer opposite effects [21], underscoring the futility of attempting to determine function in silico rather than experimentally.

Typically, human geneticists discover genotypephenotype associations based on assumptions about the functional consequences of genetic variation, and laboratory biologists pursue the mechanisms underlying these associations using relevant experimental systems. But there are important limitations of this paradigm, including 1) that small and under-studied populations are disadvantaged because findings from the best-powered association studies (usually of European-ancestry subjects) are inevitably prioritized, 2) genetic diversity can hamper discovery because associations are most easily found in homogenous populations where risk is driven by a few common variants rather than many lessfrequent variants [60-65] and 3) initial association tests fail to account for the unpredictable consequences of most human mutations. This is especially true when protein-truncating variants are not pathogenic, and when gain-of-function and loss-of-function variants in the same gene have opposite effects on disease risk [6]. By comprehensively characterizing the functional consequences of all potentially pathogenic variants in a strong candidate locus, we sought to reverse the usual paradigm and increase our power to detect an association based on a strong mechanism-based hypothesis. The result was to demonstrate a significant enrichment among ALS patients of a particular class of variants in our candidate gene-those conferring constitutive SARM1 enzymatic activity. In addition, with the increasing use of exome sequencing for genetic diagnosis of neurodegenerative disease [66], our specific results provide immediately clinically relevant information, as well as provide a methodology for assessing the likely pathogenicity of further SARM1 variants in patients. Our results regarding naturally-occurring human variants also align closely with the findings of prior structure-function investigations of SARM1, namely the centrality of the ARM domain in SARM1 autoinhibition [21-25]. All but three of the constitutively active variants we identified lie in the ARM domain, and these exceptions are the three weakest constitutively active alleles assayed (Fig. 1). The two weakest variants were also those found in healthy controls, and we speculate that the quantitative difference between these and other variants may suggest a threshold between significant differences in in vitro activity and clinically significant differences in pathogenicity. While both of these control variants show at least 5-fold higher basal enzymatic activity than the common reference allele of SARM1 in vitro, the most common ALS patient-derived variant, V184G, has more than 26-fold higher activity (Fig. 1). Finally, we also observe that constitutively active variants do not fully account for the enrichment of rare SARM1 variants associated with ALS. While constitutively-active variants are 5.7-fold enriched, non-constitutively active rare variants are also 2.8-fold enriched in ALS patients compared to controls. Notably, our group has previously probed the allosteric modulation of SARM1 using engineered mutations that alter SARM1 activation without changing basal activity [21]. We therefore hypothesize that other classes of 
pathogenic SARM1 variants may exist that act via mechanisms other than directly disrupting enzyme autoinhibition.

Fortunately, though the number of ways to disrupt SARM1 may be myriad, for patients harboring deleterious SARM1 polymorphisms the therapeutic solution could be universal. As we have demonstrated, $\mathrm{NAD}^{+}$ hydrolase activity is required for the prodegenerative function of SARM1 [41], and that catalytic machinery is an attractive drug target. Indeed, small molecule SARM1 inhibitors are currently in development [67], and we have shown that a SARM1 dominant negative gene therapy can potently block SARM1-mediated programmed axon degeneration in mice [59]. Establishing that SARM1 inhibition is safe and effective in carriers of pathogenic SARM1 variants could provide a vital stepping stone toward the use of SARM1-directed therapeutics more generally for ALS and other diseases that involve axon degeneration.

\section{Conclusions}

In summary, we identified 12 constitutively active SARM1 variants among all 42 rare missense variants reported among 8507 ALS patients and 9671 controls. These include more than half of those that are unique to those patients or that occur in multiple patients. We also demonstrate a $>5$-fold enrichment of constitutively active variants among patients compared to controls. Expression of constitutively active ALS-associated SARM1 alleles is pro-degenerative, both in vitro in cultured neurons and in vivo in the mouse central nervous system. These results implicate rare hypermorphic SARM1 alleles as candidate genetic risk factors for ALS and other neurodegenerative conditions.

\footnotetext{
Abbreviations

AAV: Adeno-associated virus; ALS: Amyotrophic Lateral Sclerosis; ARM: HEAT/ Armadillo motif; CADPR: Cyclic adenosine diphosphate ribose; CD68: Cluster of Differentiation 68; DRG: Dorsal root ganglion; EGFP: Enhanced green fluorescent protein; MLS: Mitochondrial localization signal; MTT: 3-(4,5dimethylthiazol-2-yl)-2,5-diphenyltetrazolium bromide; NAD: Nicotinamide adenine dinucleotide; NMNAT2: Nicotinamide mononucleotide adenylyltransferase 2; TIR: Toll/Interleukin receptor; TUNEL: Terminal deoxynucleotidyl transferase dUTP nick end labeling; SAM: Sterile alpha motif; SARM1: Sterile alpha and TIR motif containing 1
}

\section{Supplementary Information}

The online version contains supplementary material available at https://doi. org/10.1186/s13024-021-00511-x.

Additional file 1. Bright field and matching fluorescent images of cultured mouse DRG neurons infected with SARM1-EGFP constructs including the human reference allele, every constitutively active SARM1 variant found in ALS patients and ten rare SARM 1 variants found in controls. The expression of EGFP in neurons infected with ALS-associated constitutively active SARM 1 variants is similar or less than EGFP expression in reference SARM1-infected neurons (compare background fluorescence to cell bodies).
Additional file 2. EGFP fluorescence intensity per cell area in cultured mouse DRG neurons infected with SARM1-EGFP constructs including every constitutively active SARM 1 variant found in ALS patients and ten rare SARM1 variants found in controls, normalized to the human reference SARM1 allele. Reduced EGFP expression in cells infected with constitutively active SARM1 alleles is likely due to $\mathrm{NAD}^{+}$depletion by SARM1 leading to impaired protein translation.

Additional file 3. The ratio of $C A D P R / N A D^{+}$levels in cultured CD1 wildtype DRG neurons (i.e. expressing endogenous SARM1) infected with human SARM1 constructs carrying rare variants identified in ALS patients. The variants are significantly more active than the reference human SARM1 allele in the presence of endogenous SARM1. This activity is dependent on the activity of the SARM1 mutant, as demonstrated by the loss of activity when a second activity-abolishing mutation, E642A, is introduced into the construct. Data are expressed relative to CADPR/NAD ${ }^{+}$ in reference SARM1-expressing neurons.

Additional file 4. Forelimb grip strength in male and female mice 3 weeks after injection with SARM1 AAV constructs.

Additional file 5. Percent area of TUNEL staining in spinal cord, and percent area of CD68 staining in sciatic nerves, two days after injecting mice with SARM1 AAV constructs.

Additional file 6. Representative images of dorsal root ganglia from mice 12 weeks post intrathecal injection with AAV constructs containing SARM1 or the ALS-associated constitutively active variant SARM1 ${ }^{V 184 G}$ fused to EGFP, demonstrating similar expression levels from the two constructs.

Additional file 7. Percent of EGFP positive dorsal root ganglia from mice intrathecally injected with AAV constructs containing the reference SARM1 allele or SARM1 $1^{V 184 G}$ fused to EGFP.

\section{Acknowledgements}

We extend our thanks to Tim Fahrner and Kelli Simburger for cloning viral constructs, to Rachel McClarney and Cassidy Menendez for assistance with mouse husbandry and administering behavioral tests, and to Alicia Neiner for processing LC-MS/MS samples. This work was supported by the Hope Center Viral Vectors Core at Washington University School of Medicine.

\section{Authors' contributions}

A.J.B, J. M and A. D designed the study. X. M performed in vitro experiments. A. S performed in vivo experiments. Y. S assisted with mass spec analysis and method development. A.J.B. analyzed the data. A.J.B., J.M. and A.D. drafted and edited the figures and manuscript. All authors read and approved the final manuscript.

\section{Authors' information}

Not Applicable

\section{Funding}

This work was supported by National Institutes of Health grants (R01NS119812 to A.J.B., A.D. and J. M, R01NS087632 to A.D. and J.M., R37NS065053 to A.D., and RF1AG013730 to J.M.).

Availability of data and materials

All data relevant to this study are contained within the article.

\section{Declarations}

Ethical approval and consent to participate

All studies were approved by the Washington University Institutional Animal Care and Use Committee.

Consent for publication

Not Applicable.

\section{Competing interests}

A.D. and J.M. are co-founders, scientific advisory board members and shareholders of Disarm Therapeutics, a wholly owned subsidiary of Eli Lilly. A.J.B. and Y.S. are consultants to Disarm Therapeutics. The authors have no other competing conflicts or financial interests. 
Received: 15 September 2021 Accepted: 17 December 2021 Published online: 06 January 2022

\section{References}

1. Al-Chalabi A. Don't keep it in the family. Nature. 2017;550(7676):S112. https://doi.org/10.1038/550S112a.

2. Gelfman S, Dugger S, de Araujo Martins Moreno C, et al. A new approach for rare variation collapsing on functional protein domains implicates specific genic regions in ALS. Genome Res. 2019. https://doi.org/10.1101/ gr.243592.118.

3. Farhan SMK, Howrigan DP, Abbott LE, et al. Exome sequencing in amyotrophic lateral sclerosis implicates a novel gene, DNAJC7, encoding a heat-shock protein. Nat Neurosci. 2019;22(12):1966-74. https://doi.org/10.1 038/s41593-019-0530-0.

4. Cirulli ET, Lasseigne BN, Petrovski $\mathrm{S}$, et al. Exome sequencing in amyotrophic lateral sclerosis identifies risk genes and pathways. Science. 2015;(80). https://doi.org/10.1126/science.aaa3650.

5. Lattante S, Doronzio PN, Marangi G, Conte A, Bisogni G, Bernardo D, et al. Coexistence of variants in TBK1 and in other ALS-related genes elucidates an oligogenic model of pathogenesis in sporadic ALS. Neurobiol Aging. 2019:84:239.e9-239.e14. https://doi.org/10.1016/j.neurobiolaging.2019.03.010.

6. Lotta LA, Mokrosiński J, Mendes de Oliveira E, et al. Human Gain-of-Function MC4R Variants Show Signaling Bias and Protect against Obesity. Cell. 2019. https://doi.org/10.1016/j.cell.2019.03.044.

7. Figley MD, DiAntonio A. The SARM1 axon degeneration pathway: control of the NAD+ metabolome regulates axon survival in health and disease. Curr Opin Neurobiol. 2020;63:59-66. https://doi.org/10.1016/j.conb.2020.02.012.

8. Gilley J, Ribchester RR, Coleman MP. Sarm1 deletion, but not WIdS, confers lifelong Rescue in a Mouse Model of severe Axonopathy. Cell Rep. 2017; 21(1):10-6. https://doi.org/10.1016/j.celrep.2017.09.027.

9. Ko KW, Devault L, Sasaki Y, Milbrandt J, DiAntonio A. Live imaging reveals the cellular events downstream of SARM1 activation. Elife. 2021;10. https:// doi.org/10.7554/eLife.71148.

10. Gerdts J, Summers DW, Sasaki Y, DiAntonio A, Milbrandt J. Sarm1-mediated axon degeneration requires both SAM and TIR interactions. J Neurosci. 2013;33(33):13569-80. https://doi.org/10.1523/JNEUROSCI.1197-13.2013.

11. Osterloh JM, Yang J, Rooney TM, et al. dSarm/Sarm1 is required for activation of an injury-induced axon death pathway. Science. 2012;(80). https://doi.org/10.1126/science.1223899.

12. Ozaki E, Gibbons L, Neto NGB, et al. SARM1 deficiency promotes rod and cone photoreceptor cell survival in a model of retinal degeneration. Life Sci Alliance. 2020. https://doi.org/10.26508/lsa.201900618.

13. Geisler S, Doan RA, Strickland A, Huang X, Milbrandt J, DiAntonio A. Prevention of vincristine-induced peripheral neuropathy by genetic deletion of SARM1 in mice. Brain. 2016;139(12):3092-108. https://doi.org/10.1093/bra in/aww251.

14. Henninger N, Bouley J, Sikoglu EM, An J, Moore CM, King JA, et al. Attenuated traumatic axonal injury and improved functional outcome after traumatic brain injury in mice lacking Sarm1. Brain. 2016;139(4):1094-105. https://doi.org/10.1093/brain/aww001.

15. Ko KW, Milbrandt J, DiAntonio A. SARM1 acts downstream of neuroinflammatory and necroptotic signaling to induce axon degeneration. J Cell Biol. 2020;219(8). https://doi.org/10.1083/JCB.201912047.

16. Sasaki Y, Kakita H, Kubota S, Sene A, Lee TJ, Ban N, et al. SARM1 depletion rescues NMNAT1-dependent photoreceptor cell death and retinal degeneration. Elife. 2020;9. https://doi.org/10.7554/eLife.62027.

17. Turkiew E, Falconer D, Reed N, Höke A. Deletion of Sarm1 gene is neuroprotective in two models of peripheral neuropathy. J Peripher Nerv Syst. 2017;22(3):162-71. https://doi.org/10.1111/jns.12219.

18. Ziogas NK, Koliatsos VE. Primary traumatic axonopathy in mice subjected to impact acceleration: a reappraisal of pathology and mechanisms with highresolution anatomical methods. J Neurosci. 2018;38(16):4031-47. https://doi. org/10.1523/JNEUROSCI.2343-17.2018

19. Huppke P, Wegener E, Gilley J, Angeletti C, Kurth I, Drenth JPH, et al. Homozygous NMNAT2 mutation in sisters with polyneuropathy and erythromelalgia. Exp Neurol. 2019;320:112958. https://doi.org/10.1016/j. expneurol.2019.112958

20. Gilley J, Mayer PR, Yu G, Coleman MP. Low levels of NMNAT2 compromise axon development and survival. Hum Mol Genet. 2019;28(3):448-58. https:// doi.org/10.1093/hmg/ddy356.
21. Figley MD, Gu W, Nanson JD, Shi Y, Sasaki Y, Cunnea $K$, et al. SARM1 is a metabolic sensor activated by an increased NMN/NAD+ ratio to trigger axon degeneration. Neuron. 2021;109(7):1118-1136.e11. https://doi.org/10.1 016/..neuron.2021.02.009.

22. Sporny M, Guez-Haddad J, Khazma T, Yaron A, Dessau M, Shkolnisky Y, et al. Structural basis for SARM1 inhibition and activation under energetic stress. Elife. 2020;9. https://doi.org/10.7554/eLife.62021.

23. Jiang Y, Liu T, Lee CH, Chang Q, Yang J, Zhang Z. The NAD+-mediated selfinhibition mechanism of pro-neurodegenerative Sarm1. Nature. 2020; 588(7839):658-63. https://doi.org/10.1038/s41586-020-2862-z.

24. Shen C, Vohra M, Zhang P, Mao X, Figley MD, Zhu J, et al. Multiple domain interfaces mediate SARM1 autoinhibition. Proc Natl Acad Sci. 2021;118(4): e2023151118. https://doi.org/10.1073/pnas.2023151118.

25. Bratkowski M, Xie T, Thayer DA, Lad S, Mathur P, Yang YS, et al. Structural and mechanistic regulation of the pro-degenerative NAD hydrolase SARM1. Cell Rep. 2020;32(5):107999. https://doi.org/10.1016/j.celrep.2020.107999.

26. Fischer LR, Culver DG, Tennant $P$, Davis AA, Wang M, Castellano-Sanchez A, et al. Amyotrophic lateral sclerosis is a distal axonopathy: evidence in mice and man. Exp Neurol. 2004;185(2):232-40. https://doi.org/10.1016/j. expneurol.2003.10.004

27. Clark JA, Southam KA, Blizzard CA, King AE, Dickson TC. Axonal degeneration, distal collateral branching and neuromuscular junction architecture alterations occur prior to symptom onset in the SOD1G93A mouse model of amyotrophic lateral sclerosis. J Chem Neuroanat. 2016; 76(Pt A):35-47. https://doi.org/10.1016/j.jchemneu.2016.03.003.

28. Gilley J, Jackson O, Pipis M, et al. Enrichment of SARM1 alleles encoding variants with constitutively hyperactive NADase in patients with ALS and other motor nerve disorders. medRxiv. 2021;2021(06):17.21258268. https:// doi.org/10.1101/2021.06.17.21258268.

29. McGill BE, Barve RA, Maloney SE, et al. Abnormal microglia and enhanced inflammation-related gene transcription in mice with conditional deletion of Ctcf in Camk2a-Cre-expressing neurons. J Neurosci. 2018;38(1):200-19. https://doi.org/10.1523/JNEUROSCI.0936-17.2017.

30. Peters OM, Lewis EA, Osterloh JM, Weiss A, Salameh JS, Metterville J, et al. Loss of Sarm1 does not suppress motor neuron degeneration in the SOD1 G93A mouse model of amyotrophic lateral sclerosis. Hum Mol Genet. 2018; 27(21):3761-71. https://doi.org/10.1093/hmg/ddy260.

31. Sasaki Y, Vohra BPS, Lund FE, Milbrandt J. Nicotinamide mononucleotide adenylyl transferase-mediated axonal protection requires enzymatic activity but not increased levels of neuronal nicotinamide adenine dinucleotide. J Neurosci. 2009;29(17):5525-35. https://doi.org/10.1523/JNEUROSCI.5469-08.2 009.

32. Mao X, Moerman AM, Lucas MM, Barger SW. Inhibition of the activity of a neuronal KB-binding factor by glutamate. J Neurochem. 1999;73(5):1851-8. https://doi.org/10.1046/j.1471-4159.1999.01851.x.

33. Sasaki Y, Engber TM, Hughes RO, Figley MD, Wu T, Bosanac T, et al. CADPR is a gene dosage-sensitive biomarker of SARM1 activity in healthy, compromised, and degenerating axons. Exp Neurol. 2020;329:113252. https://doi.org/10.1016/j.expneurol.2020.113252.

34. Chan KY, Jang MJ, Yoo BB, Greenbaum A, Ravi N, Wu WL, et al. Engineered AAVs for efficient noninvasive gene delivery to the central and peripheral nervous systems. Nat Neurosci. 2017;20(8):1172-9. https://doi.org/10.1038/ nn.4593.

35. Sasaki Y, Hackett AR, Kim S, Strickland A, Milbrandt J. Dysregulation of NAD+ metabolism induces a Schwann cell dedifferentiation program. J Neurosci. 2018;38(29):6546-62. https://doi.org/10.1523/JNEUROSCI.3304-17.2018.

36. Hunter DA, Moradzadeh A, Whitlock EL, Brenner MJ, Myckatyn TM, Wei CH, et al. Binary imaging analysis for comprehensive quantitative histomorphometry of peripheral nerve. J Neurosci Methods. 2007;166(1): 116-24. https://doi.org/10.1016/j.jneumeth.2007.06.018.

37. Chen YH, Sasaki Y, DiAntonio A, Milbrandt J. SARM1 is required in human derived sensory neurons for injury-induced and neurotoxic axon degeneration. Exp Neurol. 2021;339:113636. https://doi.org/10.1016/j. expneurol.2021.113636.

38. Karczewski KJ, Francioli LC, Tiao G, et al. Variation across 141,456 human exomes and genomes reveals the spectrum of loss-of-function intolerance across human protein-coding genes. bioRxiv. 2019. https://doi.org/10.1101/ 531210

39. van der Spek RAA, van Rheenen W, Pulit SL, Kenna KP, van den Berg LH, Veldink JH. The project MinE databrowser: bringing large-scale wholegenome sequencing in ALS to researchers and the public. Amyotroph 
Lateral Scler Front Degener. 2019;20(5-6):432-40. https://doi.org/10.1080/21 678421.2019.1606244.

40. Sievers C, Platt N, Perry VH, Coleman MP, Conforti L. Neurites undergoing Wallerian degeneration show an apoptotic-like process with annexin $\mathrm{V}$ positive staining and loss of mitochondrial membrane potential. Neurosci Res. 2003:46(2):161-9. https://doi.org/10.1016/50168-0102(03)00039-7.

41. Essuman K, Summers DW, Sasaki Y, Mao X, DiAntonio A, Milbrandt J. The SARM1 toll/Interleukin-1 receptor domain possesses intrinsic NAD+ cleavage activity that promotes pathological axonal degeneration. Neuron 2017;93(6):1334-1343.e5. https://doi.org/10.1016/j.neuron.2017.02.022.

42. Geisler S, Doan RA, Cheng GC, Cetinkaya-Fisgin A, Huang SX, Höke A, et al. Vincristine and bortezomib use distinct upstream mechanisms to activate a common SARM1-dependent axon degeneration program. JCl Insight. 2019; 4(17). https://doi.org/10.1172/jci.insight.129920.

43. Li WH, Huang K, Cai Y, Wang QW, Zhu WJ, Hou YN, et al. Permeant fluorescent probes visualize the activation of SARM1 and uncover an antineurodegenerative drug candidate. Elife. 2021;10. https://doi.org/10.7554/ elife.67381.

44. Zhao ZY, Xie XJ, Li WH, et al. A Cell-Permeant Mimetic of NMN Activates SARM1 to Produce Cyclic ADP-Ribose and Induce Non-apoptotic Cell Death. iscience. 2019. https://doi.org/10.1016/j.isci.2019.05.001.

45. Ying W, Cheruku PS, Bazer FW, Safe SH, Zhou B. Investigation of macrophage polarization using bone marrow derived macrophages. J Vis Exp. 2013;(76). https://doi.org/10.3791/50323.

46. Mueller M, Wacker K, Ringelstein EB, Hickey WF, Imai Y, Kiefer R. Rapid response of identified resident endoneurial macrophages to nerve injury. Am J Pathol. 2001;159(6):2187-97. https://doi.org/10.1016/S0002-9440(10)63 070-2.

47. Fogh I, Ratti A, Gellera C, et al. A genome-wide association meta-analysis identifies a novel locus at 17q11.2 associated with sporadic amyotrophic lateral sclerosis. Hum Mol Genet. 2014. https://doi.org/10.1093/hmg/ddt587.

48. Van Rheenen W, Shatunov A, Dekker AM, et al. Genome-wide association analyses identify new risk variants and the genetic architecture of amyotrophic lateral sclerosis. Nat Genet. 2016;48(9):1043-8. https://doi.org/1 0.1038/ng.3622.

49. Melamed Z, López-Erauskin J, Baughn MW, et al. Premature polyadenylation-mediated loss of stathmin-2 is a hallmark of TDP-43dependent neurodegeneration. Nat Neurosci. 2019;22(2):180-90. https://doi. org/10.1038/s41593-018-0293-z.

50. Klim JR, Williams LA, Limone F, Guerra San Juan I, Davis-Dusenbery BN, Mordes DA, et al. ALS-implicated protein TDP-43 sustains levels of STMN2, a mediator of motor neuron growth and repair. Nat Neurosci. 2019;22(2):16779. https://doi.org/10.1038/s41593-018-0300-4.

51. Summers DW, Milbrandt J, DiAntonio A. Palmitoylation enables MAPKdependent proteostasis of axon survival factors. Proc Natl Acad Sci U S A. 2018;115(37):E8746-54. https://doi.org/10.1073/pnas.1806933115.

52. Shin JE, Miller BR, Babetto E, Cho Y, Sasaki Y, Qayum S, et al. SCG10 is a JNK target in the axonal degeneration pathway. Proc Natl Acad Sci U S A. 2012; 109(52):E3696-705. https://doi.org/10.1073/pnas.1216204109.

53. White MA, Lin Z, Kim E, et al. Sarm1 deletion suppresses TDP-43-linked motor neuron degeneration and cortical spine loss. Acta Neuropathol Commun. 2019;7(1). https://doi.org/10.1186/s40478-019-0800-9.

54. Farhan SMK, Howrigan DP, Abbott L, et al. Enrichment of rare protein truncating variants in amyotrophic lateral sclerosis patients. bioRxiv. 2018. https://doi.org/10.1101/307835.

55. Kenna KP, Van Doormaal PTC, Dekker AM, et al. NEK1 variants confer susceptibility to amyotrophic lateral sclerosis. Nat Genet. 2016;48(9):1037-42. https://doi.org/10.1038/ng.3626.

56. Covarrubias AJ, Perrone R, Grozio A, Verdin E. NAD+ metabolism and its roles in cellular processes during ageing. Nat Rev Mol Cell Biol. 2021;22(2): 119-41. https://doi.org/10.1038/s41580-020-00313-x.

57. Lautrup S, Sinclair DA, Mattson MP, Fang EF. NAD(+) in Brain Aging and Neurodegenerative Disorders. Cell Metab. 2019;30(4):630-55. https://doi. org/10.1016/j.cmet.2019.09.001.

58. de la Rubia JE, Drehmer E, Platero JL, et al. Efficacy and tolerability of EH301 for amyotrophic lateral sclerosis: a randomized, double-blind, placebo-controlled human pilot study. Amyotroph Lateral Scler Frontotemporal Degener. 2019; 20(1-2):115-22. https://doi.org/10.1080/21678421.2018.1536152

59. Geisler S, Huang SX, Strickland A, Doan RA, Summers DW, Mao X, et al. Gene therapy targeting SARM1 blocks pathological axon degeneration in mice. J Exp Med. 2019;216(2):294-303. https://doi.org/10.1084/jem.20181040.
60. Neale BM, Rivas MA, Voight BF, Altshuler D, Devlin B, Orho-Melander M, et al. Testing for an unusual distribution of rare variants. PLoS Genet. 2011; 7(3):e1001322. https://doi.org/10.1371/journal.pgen.1001322.

61. Povysil G, Petrovski S, Hostyk J, Aggarwal V, Allen AS, Goldstein DB. Rarevariant collapsing analyses for complex traits: guidelines and applications. Nat Rev Genet. 2019;20(12):747-59. https://doi.org/10.1038/s41576-019-01 77-4.

62. Gibson G. Rare and common variants: twenty arguments. Nat Rev Genet. 2012;13(2):135-45. https://doi.org/10.1038/nrg3118.

63. Ionita-Laza I, Buxbaum JD, Laird NM, Lange C. A new testing strategy to identify rare variants with either risk or protective effect on disease. PLoS Genet. 2011;7(2):e1001289. https://doi.org/10.1371/journal.pgen.1001289.

64. Li B, Leal SM. Methods for detecting associations with rare variants for common diseases: application to analysis of sequence data. Am J Hum Genet. 2008:83(3):311-21. https://doi.org/10.1016/j.ajhg.2008.06.024

65. Guo MHH, Dauber A, Lippincott MFF, Chan YM, Salem RMM, Hirschhorn JNN. Determinants of power in gene-based burden testing for monogenic disorders. Am J Hum Genet. 2016;99(3):527-39. https://doi.org/10.1016/j.a jhg.2016.06.031

66. García J-C, Bustos R-H. The Genetic Diagnosis of Neurodegenerative Diseases and Therapeutic Perspectives. Brain Sci. 2018;8(12). https://doi.org/1 0.3390/brainsci8120222.

67. Hughes RO, Bosanac T, Mao X, Engber TM, DiAntonio A, Milbrandt J, et al. Small molecule SARM1 inhibitors recapitulate the SARM1-/- phenotype and allow recovery of a metastable Pool of axons fated to degenerate. Cell Rep. 2021;34(1):108588. https://doi.org/10.1016/j.celrep.2020.108588.

\section{Publisher's Note}

Springer Nature remains neutral with regard to jurisdictional claims in published maps and institutional affiliations.

Ready to submit your research? Choose BMC and benefit from:

- fast, convenient online submission

- thorough peer review by experienced researchers in your field

- rapid publication on acceptance

- support for research data, including large and complex data types

- gold Open Access which fosters wider collaboration and increased citations

- maximum visibility for your research: over $100 \mathrm{M}$ website views per year

At $\mathrm{BMC}$, research is always in progress.

Learn more biomedcentral.com/submissions 